\title{
Induction of gibberellin 20-oxidases and repression of gibberellin 2ß-oxidases in unfertilized ovaries of entire tomato mutant, leads to accumulation of active gibberellins and parthenocarpic fruit formation
}

\author{
Francesco Mignolli • María Laura Vidoz • \\ Lorenzo Mariotti • Lara Lombardi • \\ Piero Picciarelli
}

Received: 2 July 2014/Accepted: 23 November 2014/Published online: 4 December 2014

(C) Springer Science+Business Media Dordrecht 2014

\begin{abstract}
In tomato (Solanum lycopersicum L.), auxin and gibberellins (GAs) cross-talk plays an important role during fruit-set. The entire tomato mutant has been previously reported to carry a deletion in the coding region of the SIIAA9 gene, a member of the auxin signal repressor family Aux/IAA. In this paper, we examined the role of ENTIRE gene in controlling GAs metabolism and directing spontaneous fruit initiation and early ovary growth. It was shown that, similarly to pollinated fruits, facultative parthenocarpy in entire depends on active GA metabolism, since fruit growth is suppressed when GA biosynthesis is blocked. Analysis of endogenous GAs during the first 10 days after flower emasculation revealed that entire fruits accumulated higher amounts of active GAs $\left(\mathrm{GA}_{1}\right.$ and $\mathrm{GA}_{3}$ ) in comparison to wild type pollinated fruits, suggesting that a different GA homeostasis regulation occurs. Transcript analysis of the main GA biosynthesis genes showed that differently from unpollinated and non parthenocarpic wild type ovaries, in entire active GA flux modulation is regulated by the activation of SlGA20oxl
\end{abstract}

Electronic supplementary material The online version of this article (doi:10.1007/s10725-014-0002-1) contains supplementary material, which is available to authorized users.

F. Mignolli $(\bowtie) \cdot$ M. L. Vidoz

Instituto de Botánica del Nordeste (IBONE), Facultad de

Ciencias Agrarias, Universidad Nacional del Nordeste-

CONICET, Sargento Cabral 2131, 3400 Corrientes, Argentina

e-mail: mignollif@ virgilio.it

L. Mariotti · L. Lombardi

Department of Biology, University of Pisa, Via Ghini 13,

56126 Pisa, Italy

P. Picciarelli

Department of Agriculture, Food and Environment, University

of Pisa, Via Mariscoglio 34, 56124 Pisa, Italy and SIGA20ox2 and also by a marked reduction of GA catabolism (reduced transcription of GA $2 \beta$-oxidase genes) during the early fruit expansion phase.

Keywords Entire mutant - SIIAA9 - Fruit development · Parthenocarpy · Gibberellin metabolism · Tomato

$\begin{array}{ll}\text { Abbreviations } \\ \text { AC } & \text { Ailsa Craig } \\ \text { GA } & \text { Gibberellin } \\ \text { GC-MS/ } & \text { Gas chromatography-tandem mass } \\ \text { MS } & \text { spectrometry } \\ \text { Aux/IAA } & \text { Auxin/indoleacetic acid } \\ \text { GA20ox } & \text { GA 20-oxidase } \\ \text { GA3ox } & \text { GA } 3 \beta \text {-oxidase } \\ \text { GA2ox } & \text { GA } 2 \beta \text {-oxidase }\end{array}$

\section{Introduction}

Fruit-set is defined as the start of a developmental program that leads the conversion of a static ovary into an actively growing fruit and depends on a successful pollination and ovule fertilization. In tomato, Solanum lycopersicum L., early fruit development is characterized by an intense mitotic activity in the pericarp and placenta during the first 7-14 days after fertilization. At the end of this stage, cell divisions stop and fruit growth proceeds mostly by cell expansion for 6 or 7 weeks up to its final size (Gillaspy et al. 1993).

Coordination of fruit development relies on a complicate network of hormonal, metabolic and environmental signals that has only recently started to be untangled (Ruan et al. 
2012). Among hormones, auxin and GAs are known to have a central role during fruit development. This is sustained by the fact that both hormones promote parthenocarpic fruit growth when applied to ovaries (Vivian-Smith and Koltunow 1999; Bünger-Kibler and Bangerth 1982).

Auxin- and GA-mediated fruit growth takes place through different morphological and cellular processes and their simultaneous application to ovaries is required to obtain fruits that resemble pollinated ones in their size and shape (Serrani et al. 2007a; Ozga et al. 2002). Auxin signal has been recognized as one of the main hormonal cues responsible for driving flower and fruit development (Sundberg and Østergaard 2009). Indeed, auxin content has been shown to increase in tomato fruits after pollination and its distribution across the growing fruits suggests that seeds are most likely the site of auxin biosynthesis (Mariotti et al. 2011; Pattison and Catalá 2012).

The involvement of GAs in growing tomato fruits has been extensively reported (Mapelli et al. 1978; Bohner et al. 1988; Mariotti et al. 2011). Parthenocarpic fruit growth in pat and pat-2 tomato mutants is related to a precocious activation of GA biosynthesis (Fos et al. 2000; Olimpieri et al. 2007). Moreover, the pollination stimulus is able to activate GA metabolism while application of GA biosynthesis inhibitors determines a strong reduction of fruit growth and seed number (Koshioka et al. 1994; Serrani et al. 2007b). In tomato, the increase of endogenous GA content upon pollination is associated with the upregulation of biosynthetic genes such as SICPS (copalyldiphosphate synthase), SlGA20oxl, SlGA20ox2 and SlGA20ox3 encoding GA 20-oxidases enzymes, whereas gene expression of SlGA3ox1 and SlGA3ox2 appear basically unaffected (Rebers et al. 1999; Serrani et al. 2007b). Moreover, GA accumulation does not seem to be determined by a reduction of GA inactivation due to GA $2 \beta$-oxidases activity (Serrani et al. 2007b). The importance of GAs in fruit formation was further confirmed by silencing the GA signalling repressor, SIDELLA gene, which is known to be a repressor of fruit development in tomato (Martí et al. 2007).

It is widely documented that GAs influence their own regulatory system by negative feedback regulation of GA biosynthesis and positive feedforward regulation of GA catabolism (Hedden and Thomas 2012). However, other hormones like auxin also affect GA biosynthesis, catabolism and signalling (Weiss and Ori 2007). In Arabidopsis siliques, auxin exerts control on GA metabolism by the upregulation of AtGA20oxl, AtGA20ox2 and AtGA3oxl without affecting GA 2ß-oxidase genes expression (Dorcey et al. 2009). Similarly, in tomato ovaries, auxin treatment induces fruit-set by enhancing GA biosynthesis through GA 20-oxidases and GA $3 \beta$-oxidases gene upregulation, and by reducing GA inactivation; this supports the assumption that auxin acts prior to GAs as the early post- fertilization signal (Serrani et al. 2008). Further evidence for auxin and GA interplay is provided by a reduction of the Auxin Response Factor 7 (SlARF7) gene transcripts in transgenic tomato lines in which fertilization-independent fruit growth depends on the partial activation of the auxin and GA signalling pathways (de Jong et al. 2011).

Among the auxin signalling components, the gene family Auxin/INDOLE-3-ACETIC ACID (Aux/IAA) encodes short-lived, nuclear-localized proteins with a central role in the control of auxin response (Hagen and Guilfoyle 2002). It has been demonstrated that auxin is required to facilitate the interaction between AUX/IAAs and the F-box protein TIR1 which is part of the ubiquitin ligase complex SCF ${ }^{\text {TIR1 }}$ (Dharmasiri et al. 2005). Ubiquitination of AUX/IAAs and successive proteolysis via $26 \mathrm{~S}$ proteasome are necessary to release the AUX/IAA transcription constraint of auxin responsive genes (Worley et al. 2000). In tomato, at least 26 different $A u x / I A A$ genes have been identified up to now ( $\mathrm{Wu}$ et al. 2012). The specific spatio-temporal expression pattern of several tomato Aux/IAA genes is consistent with the idea that the encoded proteins may perform specific plant developmental functions (Audran-Delalande et al. 2012).

Molecular characterization of the entire mutant of tomato revealed a recessive point mutation within the coding sequence of the SIIAA9 gene (a member of Aux/IAA family). This mutation determines a premature stop in the sequence reading frame that would convert the IAA9 protein in a functionally defective peptide (Zhang et al. 2007). The most striking phenotype of entire is the extreme reduction in leaf complexity. Reproductive organ aberrations such as asymmetrical growth of flowers and fused sepals are also common features in this mutant. Little is known about the facultative parthenocarpic nature of entire mutant. Wang et al. (2005) suggests that IAA9 would act as an auxin signalling repressor that prevents ovary development before fertilization takes place since antisense downregulation of SIIAA9 gene in the tomato cv. Micro-Tom induces parthenocarpy. However it is not known whether this precocious fruit set is mediated by a modified GA metabolism and/or response.

In this paper we report the role of ENTIRE (SIIAA9) gene as regulator of gibberellin metabolism in tomato fruit. Indeed, parthenocarpic fruit formation in entire depends on an alternative GAs metabolism regulation that leads to a high accumulation of active gibberellins.

\section{Materials and methods}

Plant material

Seeds of entire tomato (S. lycopersicum L.) mutant (accession n. LA2922) and its genetic background cv. Ailsa 
Craig (AC, accession n. LA2838A) were obtained from the Tomato Genetic Resources Center (University of California, Davis, CA, USA). Plants were grown under standard greenhouse conditions as described by Mignolli et al. (2012). Six flowers for truss were left in order to limit fruit competition. To prevent self-pollination, flowers were emasculated at pre-anthesis stage (PA) and then manually pollinated with wild type (AC) pollen or left unpollinated. Flowers were tagged and harvested at different times. In the case of entire, only unpollinated fruits that showed parthenocarpic growth were selected for analysis.

\section{Uniconazole treatments}

Ten $\mu \mathrm{L}$ of a solution composed of $0.2 \mathrm{mM}$ uniconazole (Uni) [(E)-(RS)-1-(4-Chlorophenyl)-4,4-dimethyl-2-(1H1,2,4-triazol-1-yl) pent-1-en-3-ol; Santa Cruz Biotechnology, Dallas, TX, USA], $1 \%$ ethanol and $0.1 \%$ Tween 20 were applied on emasculated pollinated and unpollinated flowers at PA stage. Equal volume of solvent was used as mock. In order to revert the effect of uniconazole, gibberellic acid $\left(\mathrm{GA}_{3} 2 \mu\right.$ ovary $^{-1}$, Sigma-Aldrich, St. Louis, MO, USA) was applied to emasculated flowers simultaneously with the inhibitor. Fruits were collected and weighted 10 days later.

\section{Quantification of endogenous GAs}

Endogenous GAs were determined in ovaries at PA, and during the first 10 days in pollinated $\mathrm{AC}$ and parthenocarpic entire fruits. Extraction, purification and GAs determination were performed according to Mariotti et al. (2011). In brief, $0.5-1 \mathrm{~g}$ of frozen ovaries/fruits were ground in mortar adding $5 \mathrm{~mL}$ of $80 \%$ methanol, centrifuged for $10 \mathrm{~min}$ at 4,000 rpm and supernatant was collected. Extraction procedure was repeated four times. A known amount of deuterated GAs $\left(\left[17,17-{ }^{2} \mathrm{H}_{2}\right]-\mathrm{GA}_{19}\right.$, $\left[17,17-{ }^{2} \mathrm{H}_{2}\right]-\mathrm{GA}_{20}, \quad\left[17,17-{ }^{2} \mathrm{H}_{2}\right]-\mathrm{GA}_{29}, \quad\left[17,17-{ }^{2} \mathrm{H}_{2}\right]-\mathrm{GA}_{1}$, $\left[17,17-{ }^{2} \mathrm{H}_{2}\right]-\mathrm{GA}_{8},\left[17,17-{ }^{2} \mathrm{H}_{2}\right]-\mathrm{GA}_{3}$, purchased from Dr. L.N. Mander, Australian National University, Canberra, Australia) were added to the extracts, as internal standards. Extracts were first partitioned with ethyl acetate and successively purified by HPLC (Kontron, Munich, Germany) equipped with Hypersil ODS C18 column $150 \mathrm{~mm}$ long and $4.6 \mathrm{~mm}$ i.d. A linear elution gradient from 10 to $100 \%$ methanol with a rate of $1 \mathrm{~mL} \min ^{-1}$ was applied. All fractions were dried and trimethylsilylated with N,Obis(trimethylsilyl) trifluoroacetamide (BSTFA) containing $1 \%$ of trimethylchlorosilane (Pierce, Rockford, IL, USA) at $70{ }^{\circ} \mathrm{C}$ for $1 \mathrm{~h}$. Endogenous GAs quantification was accomplished by GC-MS/MS equipment Saturn 2200 quadrupole ion trap mass spectrometer coupled with a $\mathrm{CP}$ 3800 gas chromatograph (Varian analytical Instrument,
Walnut Creek, CA, USA) according to the methodology described by Mariotti et al. (2011).

RNA extraction and gene expression analysis

According to Mignolli et al. (2012), total RNA was extracted from approximately $200 \mathrm{mg}$ of fruits using the TRI Reagent ${ }^{\circledR}$ (Sigma-Aldrich, St. Louis, MO, USA) following the manufacturer's procedures. Samples were initially homogenated with $2 \mathrm{~mL}$ of TRI Reagent and the supernatant was cleaned by chloroform partitioning. RNA was then precipitated by adding isopropyl alcohol followed by the addition of $75 \%$ ethanol. RNA was finally resuspended in DEPC water. RNA was subjected to purification from contaminating DNA with the TURBO DNA free kit (Applied Biosystems/Ambion, Austin, TX, USA) and $5 \mu \mathrm{g}$ of each sample was reverse transcribed into cDNA with the High-Capacity cDNA Archive Kit (Applied Biosystems). Real Time RT-PCR was performed with an ABI PRISM 7700 Sequence Detection Systems (Applied Biosystems).

Expression analysis of GA metabolism genes and ToF$Z Y$ gene was carried out using TaqMan Universal PCR Master Mix (Applied Biosystems) in presence of $200 \mathrm{ng}$ of cDNA and specific probes as reported by Mariotti et al. (2011). Transcripts analysis of LeGAST1 was performed using $50 \mathrm{ng}$ of cDNA and Power $\mathrm{SYBR}^{\circledR}$ Green PCR master mix. Primer pairs used for LeGAST1 were: Fw 5'-ACAATGGCTGGGAAAATGAG-3' and Rv 5'-CTCTG TTGTTGCTGCTGCTC-3'.

Transcript levels of all genes were normalized with the

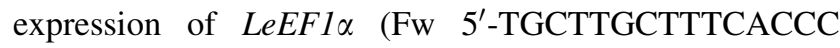
TTGGT-3', Rv 5'-CGATTTCATCATACCTAGCCTTG GA-3 TaqMan probe 5'-CTGCTGTAACAAGATGGATGC $3^{\prime}$ for TaqMan amplification method or Fw 5'-GCTGCTGT AACAAGATGGATGC-3', Rv 5'-GGGGATTTTGTCAGG GTTGTAA-3' for SYBR ${ }^{\circledR}$ Green amplification method) used as internal control. Each sample was composed of a pool of five or ten fruits and all analyses were repeated twice (Fig. 1).

Statistical methods

One-way ANOVA analysis of variance with Tuckey's post test was performed for the experiments in Fig. 2B, C using Infostat version 2012 (di Rienzo et al. 2012).

Gene accession numbers

SlGA20ox1 no. AF049898; SlGA20ox2 no. AF049899; SlGA20 ox3 no. AF04900; SlGA3oxl no. AB010991; SlGA3ox2 no. AB010992; SlGA2ox1 no. EF441351; SlGA2ox2 no. EF441352; SlGA2ox3 no. EF441353; SlGA2ox4 no. 
EF441354; SlGA2ox5 no. EF441355; LeGAST1 no. X63093; LeEF $1 \propto$ no. X53043.

\section{Results}

Spontaneous parthenocarpy in entire mutant

While no fruit-set was observed in AC unpollinated pistils, around $25 \%$ of unfertilized entire ovaries showed spontaneous and facultative parthenocarpic growth (Table 1)

Table 1 Fruit-set percentage in pollinated and unpollinated ovaries of AC and entire in three independent experiments

\begin{tabular}{llcr}
\hline & AC & \multicolumn{1}{c}{ Entire } \\
\hline Pollinated & Exp. I & $27 / 27(100 \%)$ & $16 / 16(100 \%)$ \\
& Exp. II & $14 / 14(100 \%)$ & $18 / 18(100 \%)$ \\
Unpollinated & Exp. I & $0 / 69(0 \%)$ & $24 / 115(20.9 \%)$ \\
& Exp. II & $0 / 38(0 \%)$ & $9 / 32(28.1 \%)$ \\
\hline
\end{tabular}
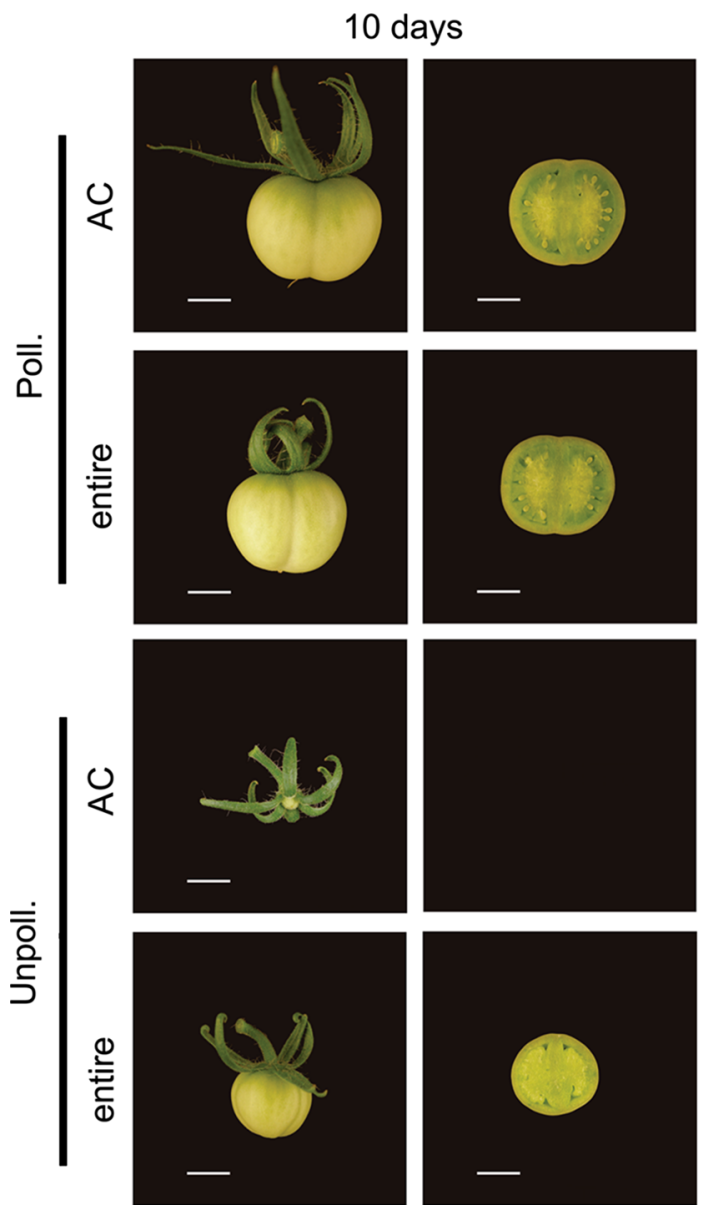
entire mutant is mediated by GA biosynthesis, we performed treatments with uniconazole (Uni), an inhibitor of the ent-kaurene oxidase (Rademacher 2000) (Fig. 2C). Application of Uni to pollinated ovaries significantly reduced fruit growth in AC and entire, although the effect was less severe in the mutant. In both genotypes,

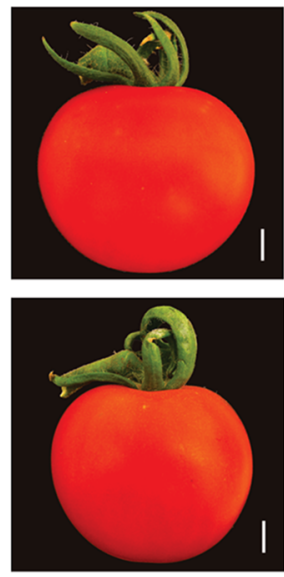

65 days

In order to ascertain whether fruit development in the

with the development of a normal locular tissue (Fig. 1). Although no differences in size were observed between pollinated AC and entire fruits at 10 and 65 days after pollination, unpollinated parthenocarpic fruits of entire were significantly smaller than pollinated ones. Indeed, parthenocarpic fruits gained weight at a slower rate than pollinated ovaries and after 10 days from emasculation their weight was $1 / 5$ of AC pollinated ones at the corresponding stage (Fig. 2A). At full ripe stage this difference was reduced and parthenocarpic fruits were $31.5 \%$ smaller than wild type pollinated ones (Fig. 2B).

Inhibition of GA biosynthesis in pollinated and unpollinated fruits
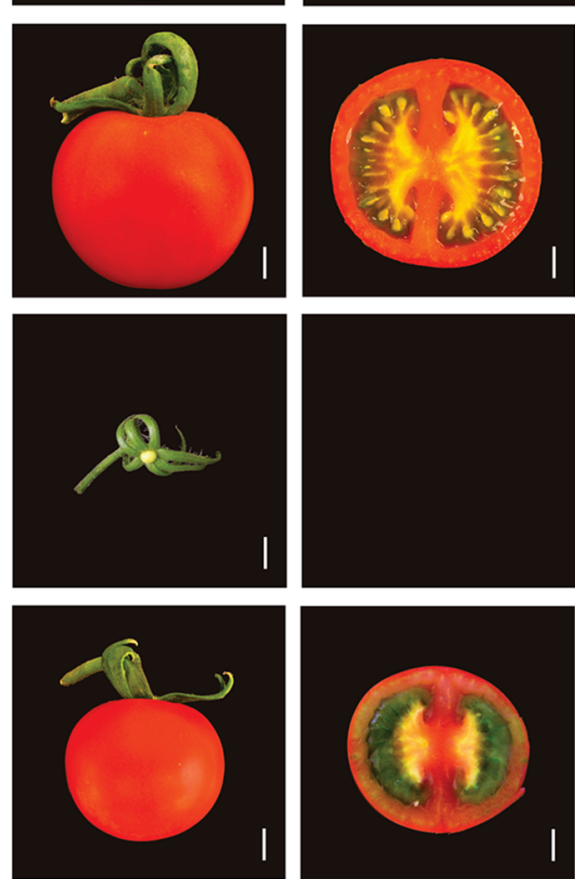

Fig. 1 Overview of pollinated and unpollinated fruits of entire and AC at 10 and 65 days (full ripe stages). Cross sections of AC and entire growing fruits are shown. White bars indicate $1 \mathrm{~cm}$ 
Fig. 2 Growth of pollinated and unpollinated $\mathrm{AC}$ and entire fruits during the first 10 days (A). In entire we selected only those fruits whose weight was higher than the maximum registered in $\mathrm{AC}$ unpollinated ovaries at the same stage. B Weight of 65-day-old fruits corresponding to full-ripe stage. C Inhibition of GA biosynthesis by uniconazole (Uni) in pollinated and unpollinated ovaries of AC and entire mutant. Fruits and ovaries were collected after 10 days from the treatment and weighed. Statistical analysis was performed in pollinated and unpollinated fruits for each genotype separately. Data are the means of 15-20 fruits. Different letters indicate statistical difference $(P<0.05)$
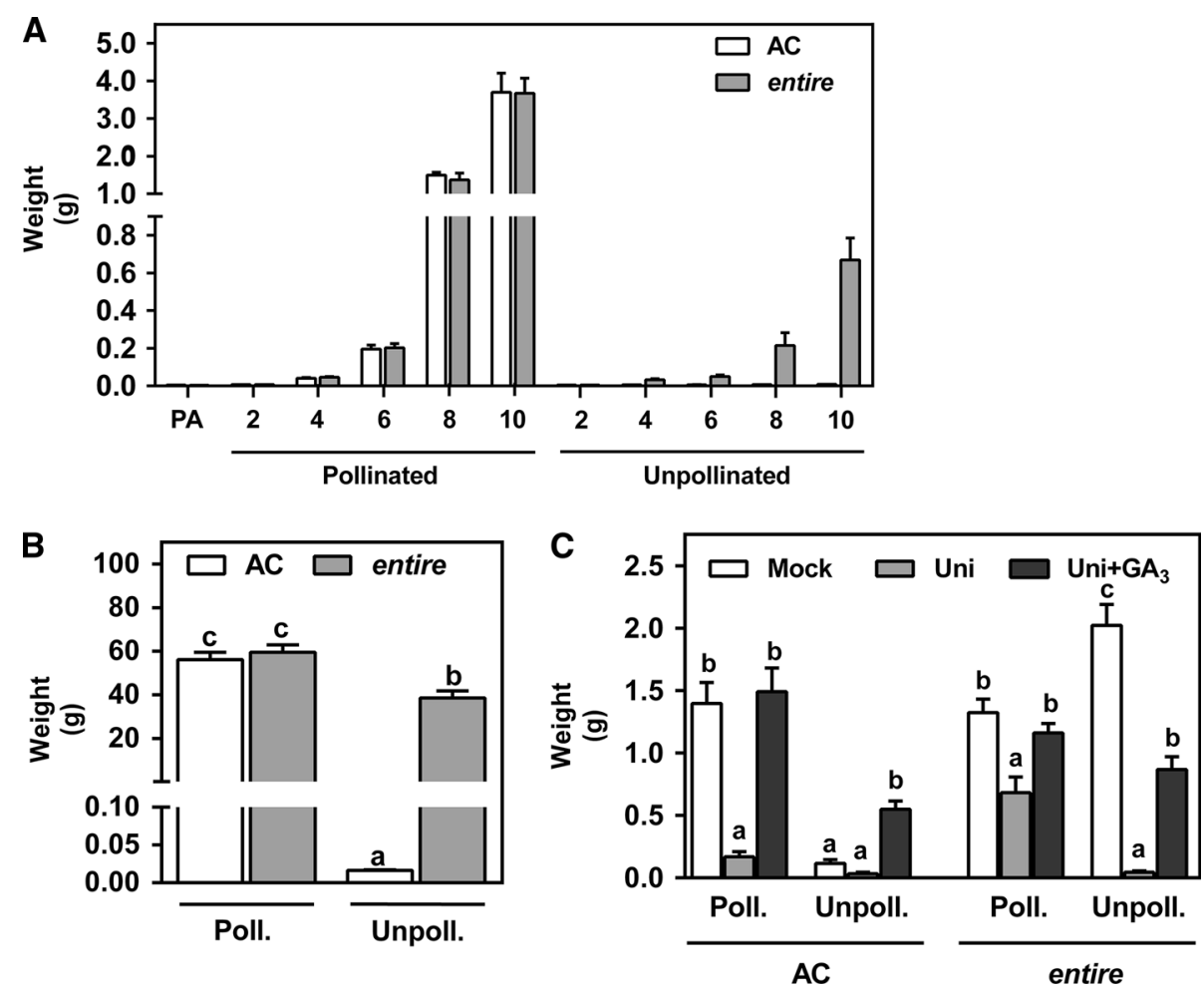

application of $\mathrm{GA}_{3}$ counteracted the effect of the inhibitor. Notably, mock-treated entire ovaries grew parthenocarpically but application of Uni strongly prevented fruits from setting. Simultaneous application of Uni and $\mathrm{GA}_{3}$ was able to revert, at least in part, the effect of the inhibitor on entire fruit growth. The difference in fruit weight observed between 10-day-old fruits in Fig. 2A and C is most likely due to the presence of ethanol in the mock solution used to treat ovaries in this experiment. Indeed, even the lowest possible ethanol concentration reduced growth in pollinated fruits, possibly due to toxic effects during fertilization, but significantly enhanced unpollinated ovary growth (data not shown). In this respect, it has been reported that alcohols exert some positive effect on vegetative growth although the precise mechanism remains controversial (Rowe et al. 1994; McGiffen and Manthey 1996).

\section{Endogenous GA levels in parthenocarpic fruits of entire}

To determine whether parthenocarpic fruit development in entire was associated to GAs accumulation, we measured the endogenous content of some GAs from the early 13-hydroxylation pathways $\left(\mathrm{GA}_{19}, \mathrm{GA}_{20}, \mathrm{GA}_{1}, \mathrm{GA}_{3} \mathrm{GA}_{29}\right.$ and $\mathrm{GA}_{8}$ ) during the first 10 days from emasculation (Fig. 3). At pre-anthesis stage GA levels did not differ substantially between AC and entire. Similarly to pollinated ovaries, concentration of $\mathrm{GA}_{19}$ (the precursor of $\mathrm{GA}_{20}$ ) progressively decreased in entire fruits, indicating that conversion into $\mathrm{GA}_{20}$ took place. In fact, the conversion rate of $\mathrm{GA}_{19}$ into $\mathrm{GA}_{20}$ was clearly reduced in entire between 4 and 10 days from emasculation as indicated by the higher $\mathrm{GA}_{19}$ to $\mathrm{GA}_{20}+\mathrm{GA}_{29}$ ratio (O'Neill et al. 2010, see supplementary data $\mathrm{S} 1$ ).

The amount of biologically active $\mathrm{GA}_{1}$ was reduced to less than a half 2 days after pollination in $\mathrm{AC}$ and slowly increased during the following stages. On the contrary, entire accumulated a five-fold higher level of $\mathrm{GA}_{1} 2$ days after emasculation and this high level was maintained throughout the first 10 days. $\mathrm{GA}_{3}$ (other biologically active GA) was also detected in tomato fruits and, similarly to $\mathrm{GA}_{1}$, its content was generally higher in entire, with the highest peak at 6 days from emasculation. The product of $2 \beta$-hydroxylation of $\mathrm{GA}_{1}$ leads to its inactive form $\mathrm{GA}_{8}$. In wild type fruits, pollination determined a marked increase of $\mathrm{GA}_{8}$ levels at 8 and 10 days from pollination. With an opposite trend, $\mathrm{GA}_{8}$ accumulated during the first 4 days from emasculation in entire fruits, to decrease afterwards down to levels that are five-fold lower than in AC. The inactive form of $\mathrm{GA}_{20}, \mathrm{GA}_{29}$, is constantly lower in entire parthenocarpic fruits from two to 10 days and peaked in $\mathrm{AC}$ fruits at 4 days.

Expression of GA metabolism genes and a LeGAST1, a GA responsive gene

To evaluate whether ENTIRE gene plays a role in regulating the expression of GA biosynthesis genes, relative 


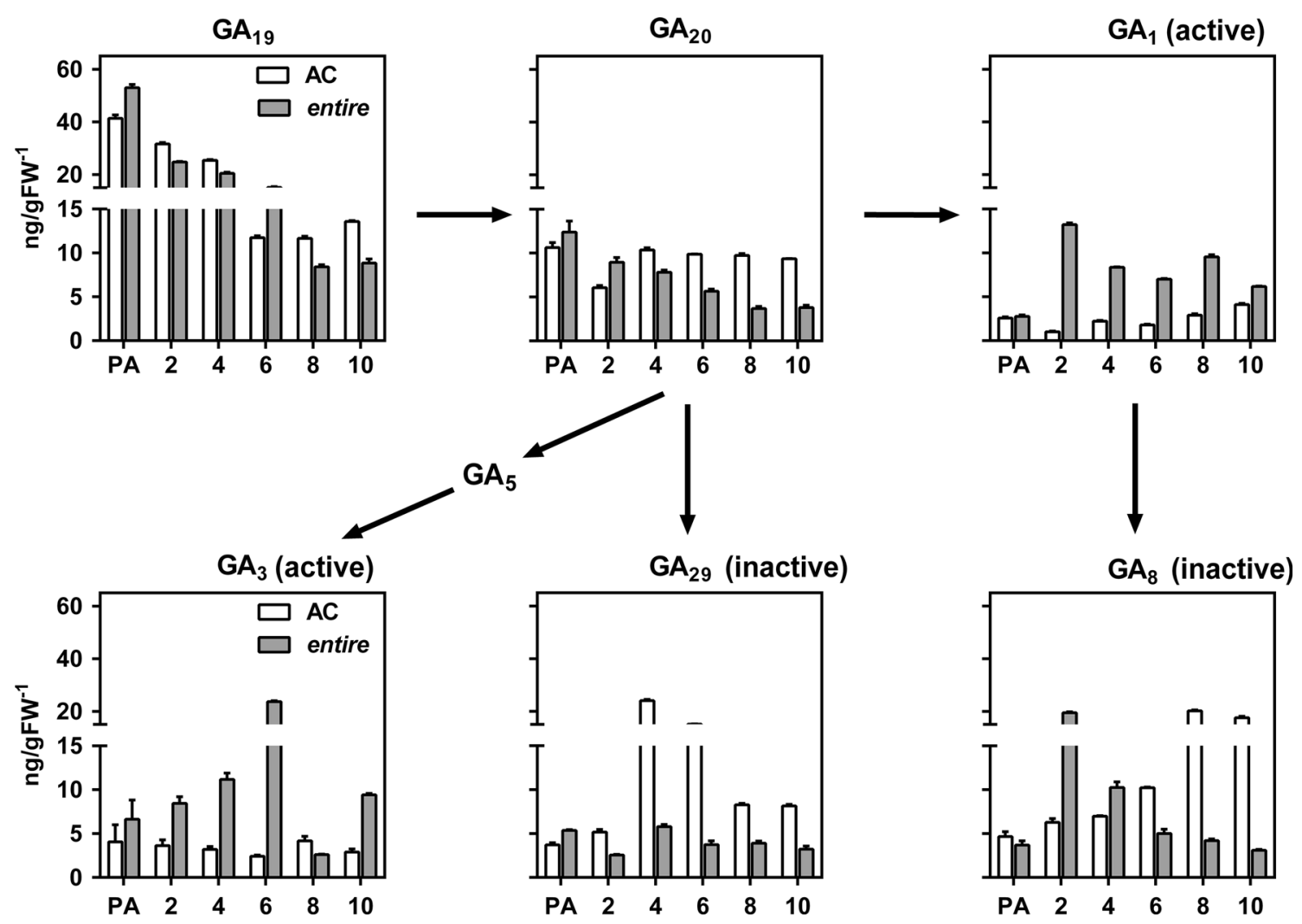

Fig. 3 Concentration of $\mathrm{GA}_{19}, \mathrm{GA}_{20}, \mathrm{GA}_{29}, \mathrm{GA}_{3}, \mathrm{GA}_{1}$ and $\mathrm{GA}_{8}$ in pollinated $\mathrm{AC}$ and parthenocarpic entire fruits at pre-anthesis stage (PA) and $2,4,6,8$ or 10 days after pollination $(\mathrm{AC})$ or emasculation (entire). Data are mean $\pm \mathrm{SE}(\mathrm{n}=3)$

transcript abundance of SlGA20oxl, SlGA20ox2, SlGA20ox3, SlGA3oxl and SlGA3ox2 was analysed in pollinated and unpollinated $\mathrm{AC}$ and entire unpollinated ovaries/fruits (Fig. 4).

Among members of the GA 20-oxidase gene family, SlGA20oxl was the most highly upregulated. Its expression in AC and entire fruits sharply increased from the time of pollination to 8 days later. While in $\mathrm{AC}$ unpollinated flowers the gene was practically unexpressed, in entire, SlGA20oxl was induced also in absence of pollination although at a lower extent with respect to pollinated fruits. SlGA20ox2 was induced in pollinated fruits and in entire unpollinated ovaries, while very low expression was observed in AC unpollinated ovaries. Transcripts levels of SlGA20ox3 peaked in AC and entire at 8 days after pollination and in $\mathrm{AC}$ emasculated flowers after 6 days.

In the case of AC and entire pollinated fruits, downregulation of SlGA3oxl occurred. Conversely, in unpollinated ovaries of entire and $\mathrm{AC}$ the gene reached its maximum expression at 2 days from emasculation and was maintained at higher levels than in pollinated fruits for up to 8 days from emasculation. SlGA3ox 2 was mainly expressed at PA but its transcripts were extremely low in both genotypes during all developing stages of pollinated and unpollinated fruits.
Active GAs homeostasis was also maintained by a fine modulation of GA inactivating enzymes (Hedden and Thomas 2012). In order to determine whether entire parthenocarpic fruit growth was also associated with an altered GA catabolism, we studied relative transcript levels of genes encoding GA $2 \beta$-oxidases (the main GA catabolic enzymes in tomato) SlGA2ox1, SlGA2ox2, SlGA2ox3, SlGA2ox4 and SlGA2ox5 (Serrani et al. 2007b) (Fig. 5). In $\mathrm{AC}$ and entire, with the exception of SlGA2oxl and SlGA2ox5, all SlGA2oxs transcripts dropped after pollination but upregulation of SlGA2ox4 and -5 was observed at later stages. With an opposite trend, expression level of SlGA2ox2 and -4 increased in entire during the first 4 days that followed emasculation whereas SlGA2ox3, -4 and -5 genes appeared strongly downregulated from the forth day on. Conversely, in AC unpollinated and non parthenocarpic ovaries with the only exception of SlGA2oxl all GA2oxs were highly induced.

With the aim to evaluate whether the higher content of active GAs correlated with the activation of GA signal in entire fruit, the expression of the tomato GA-inducible gene LeGASTl (Shi et al. 1992) was analysed. As shown in Fig. 6, LeGAST1 transcript accumulation was significantly higher at two, 6 and 8 days after emasculation in the mutant fruits. 

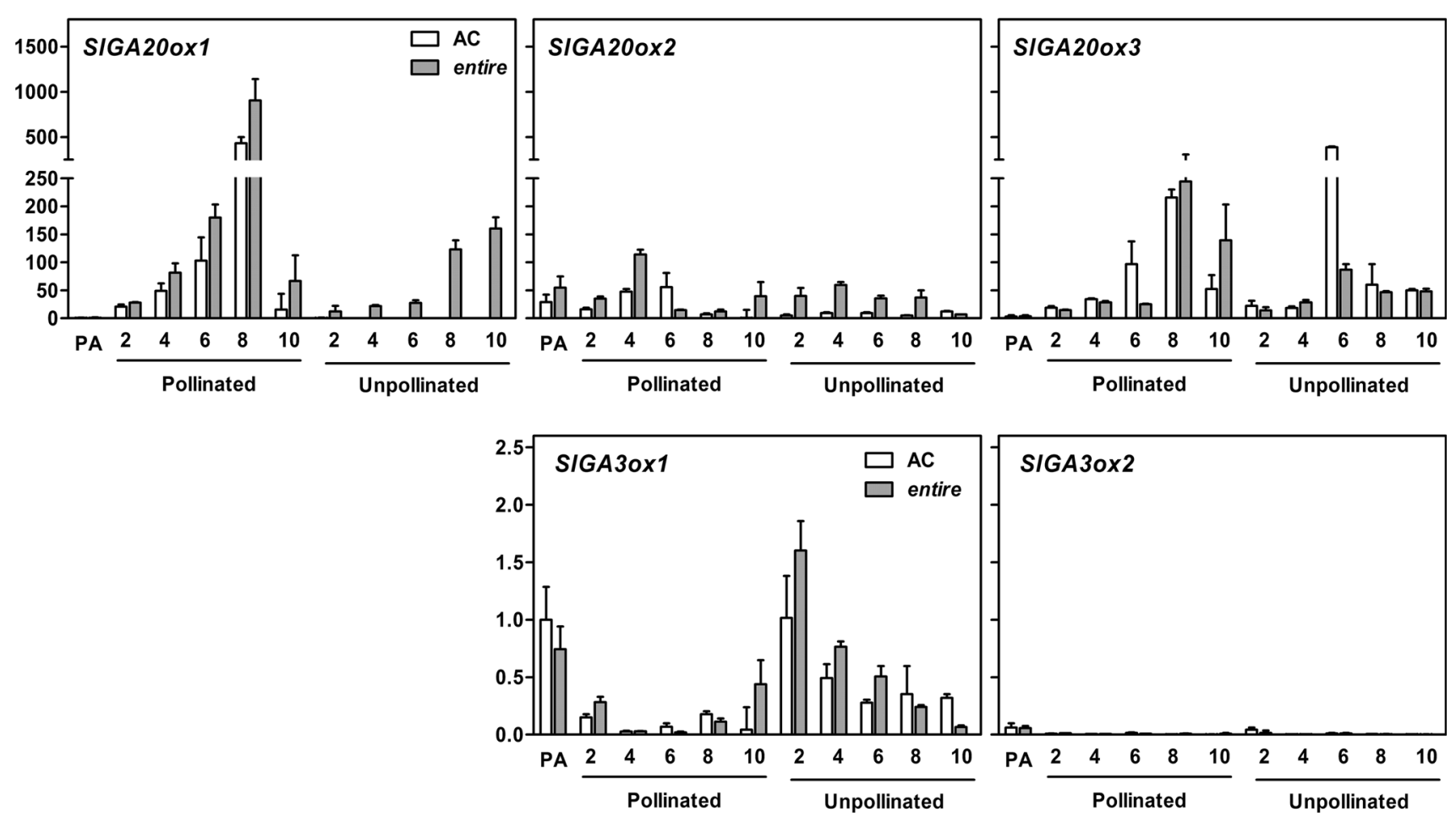

Fig. 4 Transcript levels of GA metabolism genes in pollinated AC and unpollinated parthenocarpic entire fruits. Relative expression level of SlGA20oxl, -2, and -3, SlGA3oxl, and -2 was measured during the fist 10 days from pre-anthesis stage (PA), which

\section{Discussion}

In entire tomato mutant, the lack of functionality of the auxin signalling repressor SIIAA9 (Zhang et al. 2007) leads to a strong reduction of leaf complexity as a consequence of the abnormal activation of auxin signalling in leaf margins (Koenig et al. 2009). Although this mutation also affects fruit formation, very little is known about this aspect and up to date there are no reports explaining the involvement in parthenocarpic fruit growth. Working with SIIAA9 antisense lines in Micro-Tom tomato, Wang et al. (2005) have suggested that the SIIAA9 protein would act as a negative regulator of the transition from flower to fruit in tomato. The entire mutant also showed parthenocarpy (Table 1; Fig. 1) but, differently from SIIAA9 antisense plants (Wang et al. 2009), its fruit growth rate was slower during early stages, and full ripe fruits did not reach the same size as the pollinated ones (Fig. 2A, B). In the case of entire, it is possible that these differences depend on the genetic background or on the silencing technique used by Wang et al. (2009). In this context, it should be noted that antisense SIIAA9 lines showed high variability in the level of parthenocarpy as a consequence of the degree of SIIAA9 silencing (Wang et al. 2005). Alternatively, the degree of parthenocarpy that we observed in entire together with the corresponds to the moment of emasculation and pollination (AC) or simple emasculation (entire). Expression of SlGA20oxl and SlGA3oxl at PA in AC was set to one respectively for GA20ox and GA3ox gene family. Data are mean $\pm \mathrm{SE}(\mathrm{n}=2)$

loss of domains III and IV (Zhang et al. 2007) may reveal that for SIIAA9, an independent pathway for homo- and heterodimerisation with Aux/IAA and ARF proteins (Tiwari et al. 2003) is also functioning to prevent fruit from setting. When exogenous auxin is applied to entire ovaries, a dose-dependent growth is induced (see supplementary data S2) suggesting that auxin response in fruits is not saturated by the abolishment of ENTIRE gene, and that other auxin signalling elements might be implicated to induce a full development of the fruits. Moreover, the dominance of earlier developed flowers over later developed ones in the same truss (Bangerth 1989) may play a significant role in entire parthenocarpy induction. This hypothesis is supported by the fact that when only the first flower of a truss was left in entire, the level of parthenocarpy can be raised up to $70 \%$ (data not shown) and that the highest frequency of parthenocarpic fruit growth was observed in flowers that occupy the first positions in the truss (Fig. S3).

Although auxin dynamics in the ovary have a crucial role in controlling tomato fruit development (Pattison and Catalá 2012), auxin does not act alone, as this process is largely mediated by GAs whose metabolism increases in response to auxin (Serrani et al. 2008; Ozga et al. 2009). Uniconazole application to PA flowers almost completely 

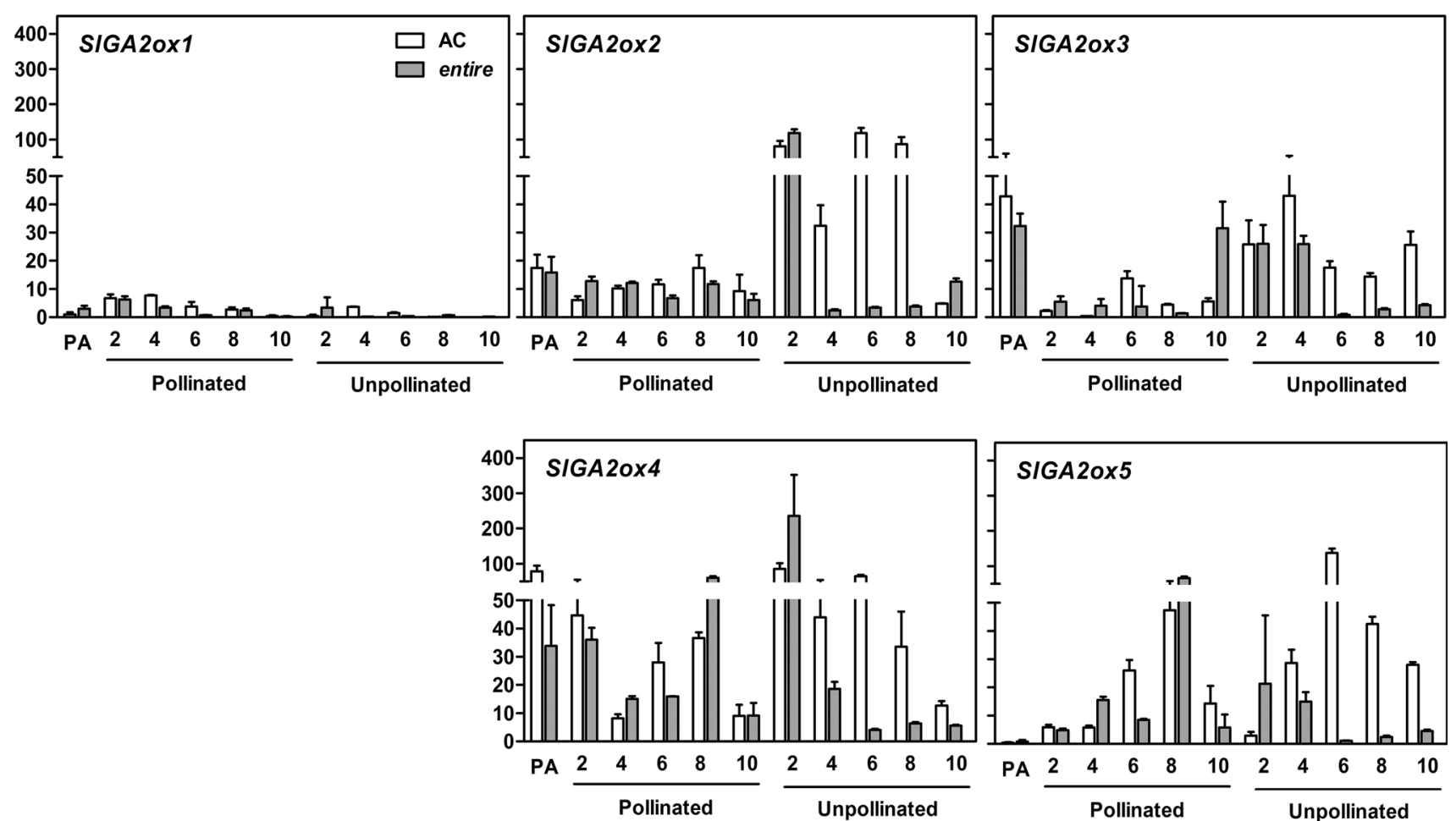

Fig. 5 Transcript level of GA catabolism genes in pollinated AC and unpollinated parthenocarpic entire fruits. Relative expression level of SlGA2oxl $,-2,-3,-4$ and -5 was studied during the first 10 days from

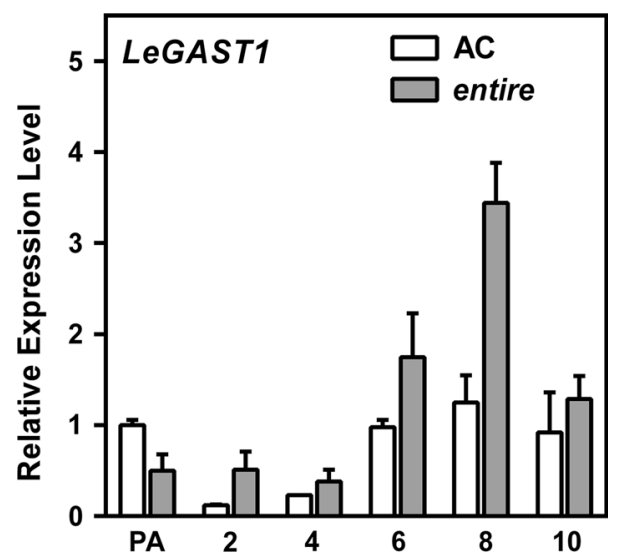

Fig. 6 GA response gene LeGAST1 in AC and entire fruits during the first 10 days from pollination (AC) or emasculation (entire). Expression at PA in AC was set to one. Data are mean $\pm \mathrm{SE}(\mathrm{n}=2)$

blocked parthenocarpic fruit growth in entire and its effect was at least partially reverted by simultaneous application of $\mathrm{GA}_{3}$ (Fig. 2C). This indicates that, similarly to pollinated fruits, fruit-set and growth of entire unfertilized ovaries depends on active metabolism of GAs.

Analysis of the endogenous level of the early 13-hydroxylation (early 13-OH) GA biosynthetic pathway, the most representative in tomato fruit (Fos et al. 2000), pre-anthesis stage (PA). Expression of SlGA2oxl at PA in AC was set to one. Data are mean $\pm \mathrm{SE}(\mathrm{n}=2)$

was performed in order to assess whether GA metabolism is affected by the mutation. In many cases, parthenocarpic fruit development was associated with accumulation of active GAs very early in ovary development (Olimpieri et al. 2007; Fos et al. 2000, 2001). Conversely, entire did not accumulate significantly higher amounts of GAs at PA in comparison to non-parthenocarpic AC ovaries (Fig. 3). Although evidently essentials for fruit growth (Fig. 2C), it is unlikely that GAs can constitute the first signal responsible for priming parthenocarpy in entire. Wang et al. (2009) have proposed that SIIAA9 would control fruit initiation by regulating photoassimilate loading into the ovary along with auxin and ethylene signalling activation. However, the participation of other hormones cannot be excluded in consideration of a possible modified auxin/GA interaction when auxin signal repression imposed by SIIAA9 is abolished (Wang et al. 2005).

The endogenous GA pattern indicates that an altered GA homeostatic regulation takes place during fruit-set and early growth in absence of a functional ENTIRE gene in unpollinated ovaries. Indeed, entire fruits exhibit a higher content of active GAs $\left(\mathrm{GA}_{1}\right.$ and $\left.\mathrm{GA}_{3}\right)$ than pollinated wild type fruits (Fig. 3). Since $\mathrm{GA}_{8}$ is the direct catabolite of $\mathrm{GA}_{1}$, its content is considered the record of the amount of $\mathrm{GA}_{1}$ that was formerly present (Coles et al. 1999). Thus, the decrease of $\mathrm{GA}_{8}$ that is registered during the early 
expansion phase in entire could be due to a slower conversion of $\mathrm{GA}_{1}$ into $\mathrm{GA}_{8}$. It should be noted that higher levels of active GAs could also be the result of the lower conversion from $\mathrm{GA}_{20}$ to $\mathrm{GA}_{29}$ which results in a higher availability of active GAs precursor (Fig. 3).

The GAST gene family is known to be rapidly induced by GAs in different plant species (Aubert et al. 1998; BenNissan and Weiss 1996; Zimmermann et al. 2010). In tomato, transcription of the LeGAST1 is positively regulated by $\mathrm{GA}_{3}$ (Shi et al. 1992; Serrani et al. 2008). The high LeGAST1 expression level found in entire provided further evidence that the elevated level of active GAs triggers the GA signal transduction (Fig. 6).

The accordance between endogenous GA levels and transcript abundance of GA biosynthesis and catabolism genes supports the general use of gene expression analysis to infer protein levels and enzymatic activity (Nadeau et al. 2011). In our work, we analysed the expression of GA 20 -oxidases and GA $3 \beta$-oxidases genes in both pollinated $\mathrm{AC}$ and parthenocarpic entire fruits. Gibberellin C-20 oxidation is considered a rate-limiting step for the regulation of active GAs flux in fruits (Rebers et al. 1999; Serrani et al. 2007b). In this sense, parthenocarpy in pat tomato mutant is associated with higher accumulation of GAs as a consequence of high SlGA20oxl induction (Olimpieri et al. 2007). While SlGA20oxl was not expressed in AC in absence of pollination, it was induced in entire unpollinated fruits at a lower extent than in pollinated fruits (Fig. 4). It is then conceivable that the partial activation of auxin signalling in entire would be sufficient to induce SlGA20oxl in growing fruits. It is known that SlGA20oxl is induced by auxin probably due to the presence of auxin responsive elements in its promoter region (Martí et al. 2010). These findings are supported by the role of auxin in enhancing GA 20-oxidases gene expression as previously reported in Arabidopsis siliques (Dorcey et al. 2009), pea pericarps (Ngo et al. 2002) and tomato fruits (Serrani et al. 2008). Since SlGA20oxl transcript levels was higher in pollinated entire fruits than in parthenocarpic fruits, it suggests that other fertilization-linked signals could exert a supplemental inductive effects on SlGA20oxl gene.

In the last step, active GAs are produced by the action of a class of 2-oxoglutarate dependent dioxygenases, GA $3 \beta$ oxidases, that convert $\mathrm{GA}_{20}$ and $\mathrm{GA}_{9}$ to $\mathrm{GA}_{1}$ and $\mathrm{GA}_{4}$, respectively. $\mathrm{GA}_{3}$ is also synthesized from $\mathrm{GA}_{20}$ through $\mathrm{GA}_{5}$ as intermediate (Hedden and Thomas 2012). In tomato, SlGA3oxl was barely transcribed early after pollination in both genotypes. Nevertheless, high transcript levels of SIGA3oxl in entire parthenocarpic fruits during the first 6 days from emasculation (Fig. 4) are probably associated with the lack of a fertilization signal, since the gene is also expressed in AC unpollinated and non parthenocarpic ovaries.

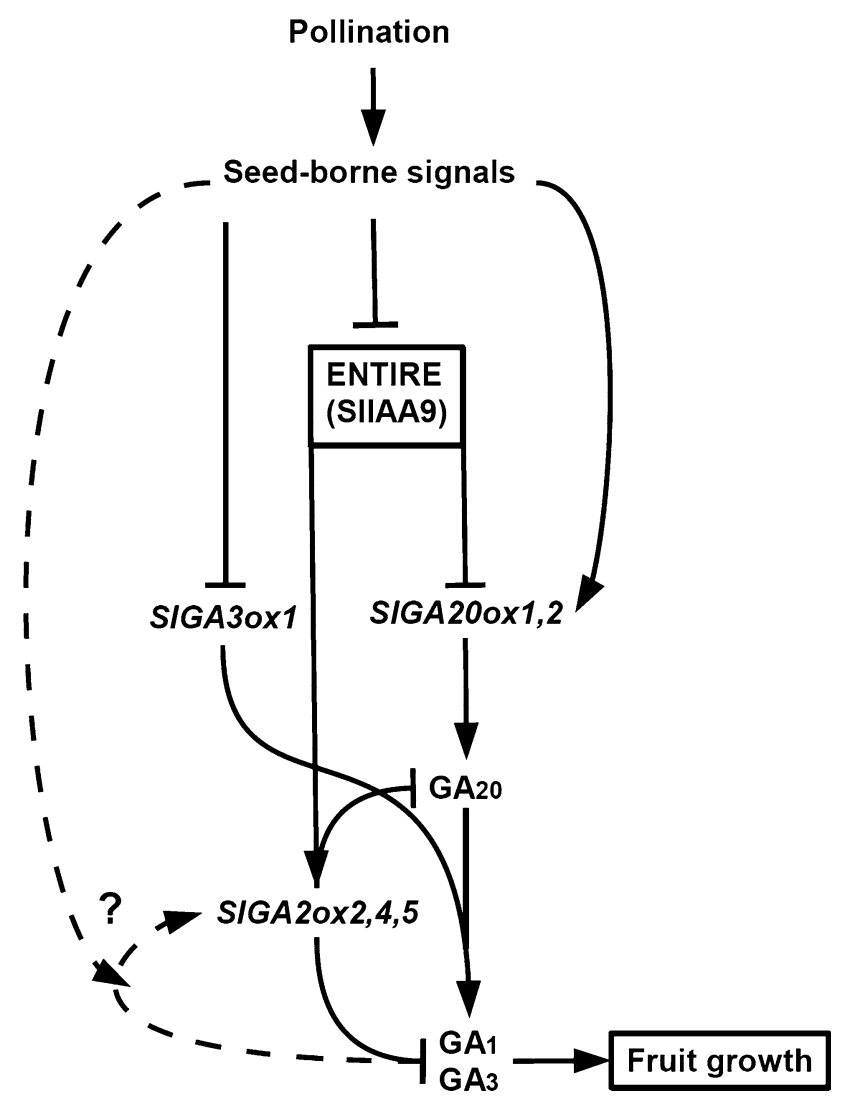

Fig. 7 Schematic model for the proposed role of ENTIRE (SIIAA9) protein in the regulation of gibberellin metabolism during fruit development. When pollination takes place, seed-specific signals degrade ENTIRE (IAA9) protein as reported by Wang et al. (2009). Release of IAA9 repression plus other seed-borne signals induces GA biosynthesis by strong activation of GA 20-oxidases genes (mostly SlGA20ox1). Homeostatic levels of active GAs are in turn controlled by a pollination-dependent feed-forward regulation (?) of GA2oxs (segmented lines) that cause the inactivation of $\mathrm{GA}_{20}$ and $\mathrm{GA}_{1}$. In absence of fertilized ovules, impaired function of the ENTIRE gene results in the accumulation of active gibberellins $\left(\mathrm{GA}_{1}\right.$ and $\left.\mathrm{GA}_{3}\right)$ through the combined action of GA 20-oxidases (GA20ox1, -2) and by downregulation of GA2oxs genes $(G A 20 x 2,-4,-5)$ that reduces GA catabolism

Endogenous GAs homeostasis not only depends on biosynthetic processes but also on reactions that lead to GA inactivation (Thomas et al. 1999). It is well documented that negative feedback regulation of GA biosynthesis and positive feed-forward control of GA catabolism enzymes (particularly GA $2 \beta$-oxidases) are the two mechanisms that maintain active GAs within physiological limits (Hedden and Thomas 2012). Consistent with this, high content of $\mathrm{GA}_{8}$ and strong upregulation of SlGA2ox4 and -5 genes (Fig. 5) were observed in pollinated fruits in both genotypes and paralleled with the induction of SIGA20oxl. Interestingly, in unpollinated wild type ovaries, where it is known that both metabolism and accumulation of GAs are extremely reduced (Koshioka et al. 1994; Serrani et al. 
2008; Mariotti et al. 2011), the high induction of most GA2oxs seems independent from the feed-forward regulation. In entire unpollinated fruits that showed high active GAs content, most of GA2oxs were found downregulated after 6 days from emasculation but were upregulated in entire pollinated fruits (Fig. 5), suggesting that a positive feed-forward regulation is only active in response to pollination.

In summary, mutation of ENTIRE (SIIAA9) gene determines the release of at least part of the auxin signal that was sufficient to circumvent the pollination stimulus and initiate fruit growth in a facultative way. Our results suggest that the ENTIRE protein could participate in GAs metabolism by inhibiting SlGA20oxl and SlGA20ox2 but activating most of GA $2 \beta$-oxidases in absence of pollination (see scheme in Fig. 7). Indeed, knock out of ENTIRE gene leads to high accumulation of active GAs as response to high activity of SlGA3oxl in presence of a basal induction of SlGA20oxl. At later stages the concerted action of SlGA20oxl upregulation and the strong reduction of GA catabolism would contribute to maintain the higher levels of active GAs at the start of expansion phase in entire parthenocarpic fruits. In presence of fertilization stimulus the inactivation of ENTIRE protein (Wang et al. 2009) takes place. This, that along with other signals, produces a high induction of SlGA20oxl concomitantly with the increase of active GAs flux at the onset of rapid fruit growth (from 6 to 10 days). GA $3 \beta$-oxidation shows a significant reduction but does not appear to be limiting, as active GAs were still synthesized. In addition, high levels of $\mathrm{GA}_{29}$ and $\mathrm{GA}_{8}$ indicate that active GAs are subjected to a fast turnover in growing fruits following a feed-forward regulation in the pollinated wild type fruit probably as a consequence of SlGA2ox4 and SlGA2ox5 upregulation.

Acknowledgments We would like to thank Dr. A. Pardossi for providing greenhouse facilities and helpful advice during the plant growing season. We are also grateful to Dr. N. Ceccarelli for his critical remarks about the experimental design.

\section{References}

Aubert D, Chevillard M, Dorne AM, Arlaud G, Herzog M (1998) Expression patterns of GASA genes in Arabidopsis thaliana: the GASA4 gene is up-regulated by gibberellins in meristematic regions. Plant Mol Biol 36:871-883

Audran-Delalande C, Bassa C, Mila I, Regad F, Zouine M, Bouzayen $M$ (2012) Genome-wide identification, functional analysis and expression profiling of the Aux/IAA gene family in tomato. Plant Cell Physiol 53:659-672

Bangerth F (1989) Dominance among fruits/sinks and the search for a correlative signal. Physiol Plantarum 76:608-614

Ben-Nissan G, Weiss D (1996) The petunia homologue of tomato gast1: transcript accumulation coincides with gibberellininduced corolla cell elongation. Plant Mol Biol 32:1067-1074
Bohner J, Hedden P, Bora-Haber E, Bangerth F (1988) Identification and quantitation of gibberellins in fruits of Lycopersicon esculentum, and their relationship to fruit size in L. esculentum and L. pimpinellifolium. Physiol Plantarum 73:348-353

Bünger-Kibler S, Bangerth F (1982) Relationship between cell number, cell size and fruit size of seeded fruits of tomato (Lycopersicon esculentum Mill.), and those induced parthenocarpically by the application of plant growth regulators. Plant Growth Regul 1:143-154

Coles JP, Phillips AL, Crocker SJ, García-Lepe R, Mervyn JL, Hedden P (1999) Modification of gibberellin production and plant development in Arabidopsis by sense and antisense expression of gibberellin 20-oxidase genes. Plant J 17:547-556

de Jong M, Wolters-Arts M, García-Martínez JL, Mariani C, Vriezen WH (2011) The Solanum lycopersicum auxin response factor 7 (SlARF7) mediates cross-talk between auxin and gibberellin signalling during tomato fruit set and development. J Exp Bot 62:617-626

Dharmasiri N, Dharmasiri S, Estelle M (2005) The F-box protein TIR1 is an auxin receptor. Nature 435:441-445

di Rienzo JA, Casanoves F, Balzarini MG, Gonzalez L, Tablada M, Robledo CW. InfoStat versión 2012. InfoStat Group, Facultad de Ciencias Agropecuarias, Universidad Nacional de Córdoba, Argentina. http://www.infostat.com.ar

Dorcey E, Urbez C, Blázquez MA, Carbonell J, Perez-Amador MA (2009) Fertilization-dependent auxin response in ovules triggers fruit development through the modulation of gibberellin metabolism in Arabidopsis. Plant J 58:318-332

Fos M, Nuez F, García-Martínez JL (2000) The gene pat-2, which induces natural parthenocarpy, alters the gibberellin content in unpollinated tomato ovaries. Plant Physiol 122:471-480

Fos M, Proaño K, Nuez F, García-Martínez JL (2001) Role of gibberellins in parthenocarpic fruit development induced by the genetic system pat-3/pat-4 in tomato. Physiol Plantarum 111:545550

Gillaspy G, Ben-David H, Gruissem W (1993) Fruits: a developmental perspective. Plant Cell 5:1439-1451

Hagen G, Guilfoyle TJ (2002) Auxin-responsive gene expression: genes, promoters and regulatory factors. Plant Mol Biol 49:373385

Hedden P, Thomas SG (2012) Gibberellin biosynthesis and its regulation. Biochemical J 444:11-25

Koenig D, Bayer E, Kang J, Kuhlemeier C, Sinha N (2009) Auxin patterns Solanum lycopersicum leaf morphogenesis. Development 136:2997-3006

Koshioka M, Nishijima T, Yamazaki H, Liu Y, Nonaka M, Mander LN (1994) Analysis of gibberellins in growing fruits of Lycopersicon esculentum after pollination or treatment with 4-chlorophenoxyacetic acid. J Hortic Sci 69:171-180

Mapelli S, Frova C, Torti G, Soressi GP (1978) Relationship between set, development and activities of growth regulators in tomato fruits. Plant Cell Physiol 19:1281-1288

Mariotti L, Picciarelli P, Lombardi L, Ceccarelli N (2011) Fruit-set and early fruit growth in tomato are associated with increases in indoleacetic acid, cytokinin, and bioactive gibberellin contents. J Plant Growth Regul 30:405-415

Martí C, Orzáez D, Ellul P, Moreno V, Carbonell J, Granell A (2007) Silencing of DELLA induces facultative parthenocarpy in tomato fruits. Plant J 52:865-876

Martí E, Carrera E, Ruiz-Rivero O, García-Martínez JL (2010) Hormonal regulation of tomato gibberellin 20-oxidase 1 expressed in Arabidopsis. J Plant Phisiol 167:1188-1196

McGiffen ME, Manthey JA (1996) The role of methanol in promoting plant growth: a current evaluation. Hort Sci 31:1092-1096

Mignolli F, Mariotti L, Lombardi L, Vidoz ML, Ceccarelli N, Picciarelli P (2012) Tomato fruit development in the auxin- 
resistant $d g t$ mutant is induced by pollination but not by auxin treatment. J Plant Physiol 169:1165-1172

Nadeau CD, Ozga JA, Kurepin LV, Jin A, Pharis RP, Reinecke DM (2011) Tissue-specific regulation of gibberellin biosynthesis in developing pea seeds. Plant Physiol 156:897-912

Ngo P, Ozga JA, Reinecke DM (2002) Specificity of auxin regulation of gibberellin 20-oxidase expression in pea pericarp. Plant Mol Biol 49:439-448

O'Neill DP, Davidson SE, Clarke VC, Yamauchi Y, Yamaguchi S, Kamiya Y, Reid JB, Ross JJ (2010) Regulation of the gibberellin pathway by auxin and DELLA proteins. Planta 232:1141-1149

Olimpieri I, Siligato F, Caccia R, Mariotti L, Ceccarelli N, Soressi GP, Mazzucato A (2007) Tomato fruit set driven by pollination or by the parthenocarpic fruit allele are mediated by transcriptionally regulated gibberellin biosynthesis. Planta 226:877-888

Ozga JA, van Huizen R, Reinecke DM (2002) Hormone and seedspecific regulation of pea fruit growth. Plant Physiol 128:13791389

Ozga JA, Reinecke DM, Ayele BT, Ngo P, Nadeau C, Wickramarathna AD (2009) Developmental and hormonal regulation of gibberellin biosynthesis and catabolism in pea fruit. Plant Physiol 150:448-462

Pattison RJ, Catalá C (2012) Evaluating auxin distribution in tomato (Solanum lycopersicum) through an analysis of the PIN and AUX/LAX gene families. Plant J 70:585-598

Rademacher W (2000) Growth retardants: effect on gibberellin biosynthesis and other metabolic pathways. Annu Rev Plant Physiol Plant Mol Biol 51:501-531

Rebers M, Kaneta T, Kawaide H, Yamaguchi S, Yang YY, Imai R, Sekimoto H, Kamiya Y (1999) Regulation of gibberellin biosynthesis genes during flower and early fruit development of tomato. Plant J 17:241-250

Rowe RN, Farr DJ, Richards BA (1994) Effects of foliar and root application of methanol and ethanol on the growth of tomato plants (Lycopersicum esculentum Mill.). New Zeal J Crop Hort 22:335-337

Ruan YL, Patrick JW, Bouzayen M, Osorio S, Fernie AR (2012) Molecular regulation of seed and fruit set. Trends Plant Sci 17:656-665

Serrani JC, Fos M, Atarés A, García-Martínez JL (2007a) Effect of gibberellin and auxin on parthenocarpic fruit growth induction in the cv Micro-Tom of tomato. J Plant Growth Regul 26:211-221

Serrani JC, Sanjuán R, Ruiz-Rivero O, Fos M, García-Martínez JL (2007b) Gibberellin regulation of fruit set and growth in tomato. Plant Physiol 145:246-257
Serrani JC, Ruiz-Rivero O, Fos M, García-Martínez JL (2008) Auxininduced fruit-set in tomato is mediated in part by gibberellins. Plant J 56:922-934

Shi L, Gast RT, Gopalraj M, Olszewski NE (1992) Characterization of a shoot-specific, $\mathrm{GA}_{3}$ - and $\mathrm{ABA}$-regulated gene from tomato. Plant J 2:153-159

Sundberg E, Østergaard L (2009) Distinct and dynamic auxin activities during reproductive development. Cold Spring Harb Perspect Biol 1:a001628

Thomas SG, Phillips AL, Hedden P (1999) Molecular cloning and functional expression of gibberellin 2-oxidases, multifunctional enzymes involved in gibberellin deactivation. Proc Natl Acad Sci USA 96:4698-4703

Tiwari SB, Hagen G, Guilfoyle T (2003) The roles of auxin response factor domains in auxin-responsive transcription. Plant Cell 15:533-543

Vivian-Smith A, Koltunow AM (1999) Genetic analysis of growthregulator-induced parthenocarpy in Arabidopsis. Plant Physiol 121:437-451

Wang H, Jones B, Li Z, Frasse P, Delalande C, Regad F, Chaabouni S, Latché A, Pech JC, Bouzayen M (2005) The tomato Aux/IAA transcription factor IAA9 is involved in fruit development and leaf morphogenesis. Plant Cell 17:2676-2692

Wang H, Shauer N, Usadel B, Frasse P, Zouine M, Hernould M, Latché A, Pech JC, Fernie AR, Bouzayen M (2009) Regulatory features underlying pollination-dependent and -independent tomato fruit set revealed by transcript and primary metabolite profiling. Plant Cell 21:1428-1452

Weiss D, Ori N (2007) Mechanism of cross talk between gibberellin and other hormones. Plant Physiol 144:1240-1246

Worley CK, Zenser N, Ramos J, Rouse D, Leyser O, Theologis A, Callis J (2000) Degradation of Aux/IAA proteins is essential for normal auxin signalling. Plant J 21:553-562

Wu J, Peng Z, Liu S, He Y, Cheng L, Kong F, Wang J, Lu G (2012) Genome-wide analysis of Aux/IAA gene family in Solanaceae species using tomato as a model. Mol Genet Genomics 287:295311

Zhang J, Cheng R, Xiao J, Quian C, Wang T, Li H, Ouyang B, Ye Z (2007) A single-base deletion mutation in SlIAA9 gene causes tomato (Solanum lycopersicum) entire mutant. J Plant Res 120: 671-678

Zimmermann R, Sakai H, Hochholdinger F (2010) The Gibberellic acid stimulated-like gene family in maize and its role in lateral root development. Plant Physiol 152:356-365 